\title{
Hsa_circularRNA_0079201 suppresses chondrocyte proliferation and endochondral ossification by regulating the microRNA-140-3p/SMAD2 signaling pathway in idiopathic short stature
}

\author{
XIJUAN LIU ${ }^{1}$, CHEN YAN $^{2}$, XUEQIANG DENG ${ }^{3}$ and JINGYU JIA ${ }^{3}$ \\ Departments of ${ }^{1}$ Pediatrics, ${ }^{2}$ Rheumatology and ${ }^{3}$ Orthopedics, The Second Affiliated Hospital \\ of Nanchang University, Nanchang, Jiangxi 330006, P.R. China
}

Received May 24, 2020; Accepted August 11, 2020

DOI: $10.3892 /$ ijmm.2020.4737

\begin{abstract}
Circular (circ)RNAs are an important group of non-coding RNAs involved in different pathological and physiological functions, such as longitudinal bone growth. However, the effects of an increase or decrease in circRNA expression on idiopathic short stature (ISS) remain largely unknown. The present study compared the circRNA expression patterns of patients with ISS and healthy individuals to identify differentially expressed circRNAs involved in the regulation of ISS pathogenesis and their target microRNAs (miR). Microarray analysis revealed that 145 circRNAs were differentially expressed in patients with ISS, including 83 up- and 62 downregulated circRNAs. Reverse transcription-quantitative PCR confirmed that hsa_circRNA_0079201 was increased in patients with ISS compared with that in the normal individuals, whilst hsa_circRNA_0079201 overexpression in human chondrocytes was shown to significantly suppress their proliferation, hypertrophy and endochondral ossification abilities. Luciferase reporter assays identified that circRNA_0079201 acted as an miR-140-3p sponge. In situ hybridization confirmed the co-localization of circRNA_0079201 and miR-140-3p in the human chondrocyte and neonatal femur growth plate of $\mathrm{C} 57$ mice, while rescue experiments demonstrated that miR-140-3p overexpression reversed the inhibition of human chondrocyte proliferation, hypertrophy and endochondral ossification, caused by circRNA_0079201 overexpression. Bioinformatics analysis and luciferase reporter assays revealed that SMAD2 was a potential target gene of miR-140-3p. Furthermore, overexpressing circRNA_0079201 in human chondrocytes suppressed miR-140-3p and increased SMAD2
\end{abstract}

Correspondence to: Dr Jingyu Jia, Department of Orthopedics, The Second Affiliated Hospital of Nanchang University, 1 Minde Road, Nanchang, Jiangxi 330006, P.R. China

E-mail: jiaxintong9@163.com

Key words: circular RNA, microRNA, chondrocyte, idiopathic short stature protein expression level. Taken together, chondrocyte proliferation, hypertrophy and endochondral ossification in ISS was suppressed by a novel regulatory axis consisting of the hsa_circRNA_0079201/miR-140-3p/SMAD2 pathway. The present study provided evidence that hsa_circRNA_0079201 may be a potential target for ISS therapy.

\section{Introduction}

Idiopathic short stature (ISS) is characterized by a standing height of $<2$ standard deviation scores (SDSs; equivalent to the 2.3 rd percentile or commonly regarded as the $<3$ rd percentile) for a given age, sex and population, in the absence of systemic disease, a psychological disorder, nutritional imbalance, overt hormone deficiency or chromosomal abnormality (1). The pathogenesis of ISS has been investigated for $>10$ years; however, its exact etiology remains unclear (2-5). Currently, the primary treatment for ISS is recombinant human growth hormones; however, this treatment is not effective in all children with ISS (6) and treatment responses vary considerably between studies (7-10). Therefore, the pathogenesis of ISS requires further elucidation to improve methods for treating the condition.

Longitudinal bone growth is driven by growth plates (11), that consist of three distinct zones: Resting, proliferative and hypertrophic. Cells within the resting zone are known as chondrocyte-progenitor cells, which subsequently divide into chondrocytes to form the proliferative zone. Chondrocytes then proliferate and differentiate into the hypertrophic zone, where blood vessels, osteoblasts and osteoclasts convert cartilage into bone, resulting in longitudinal bone growth (11-13). Numerous studies have shown that non-coding (nc)RNAs regulate the growth plate (14-16) and thus, may affect longitudinal bone growth. For example, it has been reported that microRNAs (miRNA/miR) could regulate the endochondral ossification of the growth plate and longitudinal bone growth $(17,18)$.

Circular (circ)RNAs are an identified group of ncRNAs, that are widely expressed and highly conserved in mammalian cells (19-21). circRNAs are highly stable compared with that in other non-coding miRNAs and long ncRNAs (lncRNAs), due to their closed circular structure (19-21). Several studies 
have shown that circRNAs could regulate cell proliferation, differentiation and apoptosis in different types of cells, such as chondrocytes, tumor cells, cardiomyocytes and endothelial cells (22-26), whilst their increased or decreased expression levels has been associated with a number of diseases, including cancer, diabetes, pre-eclampsia, osteoarthritis, and cardiovascular and neurological diseases (23-26). However, to the best of our knowledge, the role of circRNAs in ISS pathogenesis has not yet been addressed.

The present study compared the circRNA expression patterns of patients with ISS and healthy individuals to identify differentially expressed circRNAs that regulate ISS pathogenesis and their target miRNAs.

\section{Materials and methods}

Study subject characteristics. A total of 152 patients with ISS and age-/sex-matched control individuals were recruited from The Second Affiliated Hospital of Nanchang University, China. Patients exhibiting the following were included in the study: i) A small-for-gestational age (birth weight or length $<10$ th percentile) during their neonatal period; ii) hormonal abnormalities [e.g. growth hormone $(\mathrm{GH})$ deficiency, with a peak level of $\leq 6 \mathrm{ng} / \mathrm{ml}$ ] or pubertal or thyroid disorders; iii) exposure to chronic conditions and environmental factors that influence human growth, such as nephrotic syndrome and inflammatory bowel disease; iv) skeletal anomalies or dysmorphic features; and/or v) cytogenetically detected chromosomal aberrations, such as chondrodysplasia and Down's syndrome; and vi) whole exon sequencing. A total of 152 blood samples were obtained from the study participants between October 2016 and March 2018, and were immediately frozen in liquid nitrogen, before storage at $-80^{\circ} \mathrm{C}$ until further analysis. For circRNA microarray analysis, 4 specimen pairs were used, whilst all 76 pairs were used to validate circRNA expression using reverse transcription-quantitative PCR (RT-qPCR). Human specimen collection was approved by the Human Research Ethics Committee of the Second Affiliated Hospital of Nanchang University. As all the participants were under the age of 12 years, written informed consent was provided by their parents or legal guardians before their enrollment.

Sample labeling and hybridization for microarray. The 4 blood sample pairs were used for microarray analysis, with the Arraystar Human circRNA microarray v2 (Arraystar, Inc.). The RNA was extracted from the samples using TRIzol $^{\circledR}$ (Thermo Fisher Scientific, Inc.) and were prepared for microarray hybridization using an Eastep ${ }^{\circledR}$ Super RNA simple total RNA kit (Promega Corporation) according to the manufacturer's protocol. Total RNA was digested using RNase R (Epicentre; Illumina, Inc.) to enrich the sample for circRNAs and eliminate linear RNAs. The circRNA samples were amplified by PCR and transcribed into fluorescent cy3-UTP-labeled cRNA using a random priming approach with the Arraystar super RNA labeling kit (Arraystar, Inc.). The total RNA concentration of each sample was measured using a NanoDrop ND-1000 Spectrophotometer (Nanodrop Technologies; Thermo Fisher Scientific, Inc.). Labeled cRNA samples were hybridized onto the Arraystar human circRNA array v2 (8x15 K; Arraystar, Inc.). After washing the slides using gene expression wash buffer 1 (cat. no. 5188-5325; Agilent Technologies, Inc.), reactions were performed using an Agilent G2505C microarray scanner (Agilent Technologies, Inc.).

Microarray analysis of differentially expressed circRNAs. To obtain raw expression data, the array images were processed using Agilent feature extraction software (v11.0.1.1; Agilent Technologies, Inc.). circRNA expression values were quantile normalized and analyzed using the Quantile algorithm and 3.11 version of limma packages (https://www. bioconductor.org/packages/release/bioc/html/limma.html) in R 3.6.0 software (https://www.R-project.org/). The obtained circRNA expression profiles were divided into ISS and control groups. Volcano plot filtering was used to identify circRNAs, that were significantly differently expressed between the ISS and control groups, and the Benjamini-Hochberg method was used to determine the false discovery rate (FDR)-corrected P-value. Statistical significance was set at a fold-change (FC) value of $>2.0$ and FDR-corrected $\mathrm{P}<0.05$. circRNA expression patterns between the two sample groups were distinguished using hierarchical clustering, while scatter plots were used to assess significantly differentially expressed circRNAs between the two groups, both using the R program.

Bioinformatics and in silico analyses. As miRNAs play significant roles in regulating longitudinal bone growth and circRNAs have been found to act as miRNA sponges by altering gene expression levels (19-21,27), the CircInteractome database (http://circinteractome.nia.nih.gov/) was used to predict the target genes of circRNAs. In addition, the miR-140-3p target genes were predicted using Starbase database (http://starbase. sysu.edu.cn/) and KEGG pathway analyses was performed using the Database for Annotation, Visualization and Integrated Discovery (DAVID; v6.8; https://david.ncifcrf. gov/). The signaling pathways were automatically identified and ranked in the webpage of DAVID according to $\mathrm{P}<0.05$ and enrichment factor.

$R T-q P C R$ validation of key circRNAs. Total RNA was extracted from the frozen specimens using an Eastep ${ }^{\circledR}$ Super RNA simple total RNA kit (Promega Corporation). After cDNA synthesis (PrimeScript $^{\mathrm{TM}}$ RT reagent kit and PrimeScript ${ }^{\mathrm{TM}}$ RT reagent kit with gDNA Eraser; Takara Bio, Inc.), circRNA expression levels were quantified using RT-qPCR with the primer sequences in Table I, and an ABI Q6 PCR system (Applied Biosystems; Thermo Fisher Scientific, Inc.). The RT-qPCR protocol used for circRNA-0079201 (TB Green ${ }^{\circledR}$ Premix Ex Taq ${ }^{\mathrm{TM}}$ II; Takara Bio, Inc.) was as follows: Initial denaturation at $95^{\circ} \mathrm{C}$ for $10 \mathrm{~min}$, followed by 40 cycles of $95^{\circ} \mathrm{C}$ for $10 \mathrm{sec}$, and $60^{\circ} \mathrm{C}$ for $34 \mathrm{sec}$. GAPDH was used as the internal control. Relative mRNA expression levels of the target genes were calculated using the $2^{-\Delta \Delta C q}$ method (28) and normalized against GAPDH, an internal control used to reduce errors, due to differences in RNA concentration and transcription efficiency. IQCE is the host gene of hsa_circRNA_0079201. For verifying the upregulation of hsa_circRNA_0079201, but not linear IQCE, RNase R (Epicentre; Illumina, Inc.) was used to degrade linear RNA. 
Table I. Primer sequences used for reverse transcription-quantitative PCR of circRNA, miRNA and mRNA expression levels.

\begin{tabular}{|c|c|}
\hline Primer name & Sequence $\left(5^{\prime}-3^{\prime}\right)$ \\
\hline circRNA_0079201F & TGTGGTGCTTCAGGCAGCTT \\
\hline circRNA_0079201R & GCTTGTACACCTTCCACTGGG \\
\hline circRNA_0000423F & TTGAATTGAAATGCAGAGCCAATG \\
\hline circRNA_0000423R & GGGTGGCCTTTTCCAACTGT \\
\hline circRNA_0051239F & GGGTGGGGCCTGTAGGATA \\
\hline circRNA_0051239R & AGGACAGCATGCCCCTGATA \\
\hline circRNA_0087631F & CACAGCTGAGAAGTAGTCAGAACT \\
\hline circRNA_0087631R & AGTTGGCTCCACATCACTGG \\
\hline circRNA_0064644F & AGGTACTCAGAGGCAGGACT \\
\hline circRNA_0064644R & TAACGTATGGCGGATGAGGC \\
\hline ActinF & AGCACAGAGCCTCGCCTTTG \\
\hline ActinR & CTTCTGACCCATGCCCACCA \\
\hline $\operatorname{miR}-140-3 \mathrm{pF}$ & GGCGTACCACAGGGTAGAA \\
\hline $\operatorname{miR}-140-3 p R$ & GTCGTATCCAGTGCAGGGTCCGAGGTATTGCACTGGATACGACCCGTG \\
\hline miR-564F & GGCATAGGCACGGTGUC \\
\hline $\operatorname{miR}-564 R$ & TGGTGTCGTGGAGTCG \\
\hline $\operatorname{miR}-635 \mathrm{~F}$ & TATAGCA TATGCAGGGTG \\
\hline miR-635R & CGCATTCGGAGTGC GAGTT \\
\hline $\mathrm{U6F}$ & CTCGCTTCGGCAGCACA \\
\hline U6R & AACGCTTCACGAATTTGCGT \\
\hline SMAD2F & ATGTCGTCCATCTTGCCATT \\
\hline SMAD2R & TTTTCTTCCTGCCCATTCTG \\
\hline IHHF & GACTCATTGCCTCCCAGAACTG \\
\hline IHHR & CCAGGTAGTAGGGTCACATTGC \\
\hline RUNX2F & ACTTCCTGTGCTCCGTGCTG \\
\hline RUNX2R & TCGTTGAACCTGGCTACTTGG \\
\hline COL10F & GCAGCATTACGACCCAAGAT \\
\hline COL10R & CATGATTGAACTCCCTGAAG \\
\hline GAPDHF & GGAGCGAGATCCCTCCAAAAT \\
\hline GAPDHR & GGCTGTTGTCATACTTCTCATGG \\
\hline
\end{tabular}

F, forward; R, reverse; circ, circular; miR, microRNA.

Cell culture. Human chondrocytes were purchased from the Procell Cell Resource Center and cultured in DMEM (Gibco; Thermo Fisher Scientific, Inc.) with 10\% FBS (Gibco; Thermo Fisher Scientific, Inc.) at $37^{\circ} \mathrm{C}$ in a humidified incubator with $5 \% \mathrm{CO}_{2}$.

Hsa_circRNA_0079201 overexpression vector and miR-140-3p mimics. The hsa_circRNA_0079201 plasmid (pHBLV-CMV-Cicr-MCS-EF1-zsgreen-T2A-puro) was successfully constructed by Hanbio Biotechnology Co., Ltd. The hsa_circRNA_0079201 overexpression vector was named as overcircRNA_0079201. Chondrocytes were seeded $\left(5 \times 10^{5}\right)$ in a 6 -well plate and cultured until $80 \%$ confluence. The overcircRNA_0079201 (5 $\mu \mathrm{l})$ was then added to the 6-well plate using Lipofectamine ${ }^{\circledR} 3000$ (Invitrogen; Thermo Fisher Scientific, Inc.). An empty vector was used as the control group. The transfection efficiency of the hsa_circRNA_0079201 vector was evaluated using a fluorescence microscope and RT-qPCR. The final concentration of hsa_circRNA_0079201 plasmid was $50 \mathrm{nM}$. The miR-140-3p mimics were obtained from Hanbio Biotechnology Co., Ltd. The $5 \mu \mathrm{lmiR}-140-3 \mathrm{p}$ mimics (sense 5'-UACCACAGGGUAGAACCACGG-3' and antisense 5'-CCGUGGUUCUACCCUGUGGUA-3') or negative control (NC) (sense 5'-UUUGUACUACACAAA AGUACUG-3', and antisense 5'-CAGUACUUUUGUGUA GUACAAA-3') were transfected into the cells in a 6-well plate using Lipofectamine 2000 (Invitrogen; Thermo Fisher Scientific, Inc.) basing on the manufacturer's instructions. The transfection efficiency of the miR-140-3p mimics was evaluated using RT-qPCR. The final concentration of miR-140-3p mimics/NC was $50 \mathrm{nM}$. The cells were harvested for further experimentation $72 \mathrm{~h}$ following transfection. All the experiments were conducted independently three times.

Cell proliferation and 5'ethynyl-2'-deoxyuridine (EdU) assays. Chondrocytes transfected with overcircRNA_0079201 were seeded in a 6-well plate $\left(5 \times 10^{5}\right)$ and cultured until $70-80 \%$ confluent. The proliferation rate was assessed using a Cell 
Counting Kit-8 (CCK-8; TransGen Biotech Co., Ltd.) kit in accordance with the manufacturer's protocol. Briefly, $2 \times 10^{3}$ chondrocytes were seeded in 96-well plates and incubated at $37^{\circ} \mathrm{C}$ in a humidified incubator with $5 \% \mathrm{CO}_{2}$, and their proliferation was examined once every $24 \mathrm{~h}$, for $96 \mathrm{~h}, 4$ times in total. After $24 \mathrm{~h}, 10 \mu \mathrm{l} \mathrm{CCK}-8$ was added to each well, the cells were cultured for $2 \mathrm{~h}$ at $37^{\circ} \mathrm{C}$ in a humidified with incubator $5 \% \mathrm{CO}_{2}$, and the solution was measured spectrophotometrically at $450 \mathrm{~nm}$ (Varioskan Flash, Agilent Technologies, Inc.). For evaluating the role of circRNA_0079201 on the chondrocyte proliferation, the normal control group (empty vector) and the blank group (neither overcircRNA_0079201 or empty vector) were used as the control group in order to compare to overcircRNA_0079201 group. All statistical analyses were performed using GraphPad Prism (v5.0; GraphPad Software, Inc.) software.

Chondrocytes were incubated with EdU (Guangzhou Ribobio Co., Ltd.) for $2 \mathrm{~h}$ according to the manufacturer's instructions. The chondrocytes were then treated with $200 \mu \mathrm{l}$ $1 \mathrm{X}$ Apollo reaction mixture for $25 \mathrm{~min}$ (Cell proliferation test kit; Beijing Solarbio Science and Technology Co., Ltd.). Subsequently, Hoechst $33342(5 \mu \mathrm{g} / \mathrm{ml})$ was added to each well to stain at room temperature $\left(22 \pm 3^{\circ} \mathrm{C}\right)$ for $30 \mathrm{~min}$, following which the cells were observed using a fluorescence microscope (x4 magnification) and images were obtained.

Luciferase reporter assay. The sequence of hsa circ_0079201-wt (5'-GCCCAAGAGCTCCCAGCTCCCACT CCCAGCAGCAGGCACTGCGAGCAAGACTGGCCGCCG GATTCCAGCGAGGAGGGGCTCCCGCGGCCCCGCTCC CCCTGCTCTGATGGGAGAAGAGACGCCGCGGCCA GAGTCCTGCAGGCCCAGTGGAAGGTGTACAAGCACA AGAAAAAAAAGGCTGTTCTGGATGAGGCGGCTG TGGTGCTTCAGGCAGCTTTCAGGGGACATCTCACGC GGACAAAGCTCTTAGCAAGCAAAGCACATGGCTCAG AGCCACCCAGCGTGCCAGGCCTCCCAGACCAG-3') and the sequence of hsa_circ_0079201-mu (5'-GCCCAAGAGCT CCCAGCTCCCACTCCCAGCAGCAGGCACTGCGAGCA AGACTGGCCGCCGGATTCCAGCGAGGAGGGGCTCCC GCGGCCCCGCTCCCCCTGCTCTGATGGGAGAAGAGA CGCCGCGGCCAGAGTCCTGCAGGCCCAGTGGAAGGT GTACAAGCACAAGAAAAAAAAGGCTGTTCTGGA TGAGGCGGgTcTcGaGCTTCAGGCAGCTTTCAGGGG ACATCTCACGCGGACAAAGCTCTTAGCAAGCAAAGC ACATGGCTCAGAGCCACCCAGCGTGCCAGGCCTCCC AGACCAG-3') were added into the pSI-Check2 vector. The hsa circRNA_0079201 plasmid or its mutated fragments (Fig. S1A) and miR-140-3p mimic were co-transfected into the 293T cell line using Lipofiter (Hanbio Biotechnology Co., Ltd.) according to the manufacturer's instructions. Similarly, the sequence of SMAD2-3UTR-wt was: 5'-AGCTTCACCAATCAAGTCCCA TGAAAAGACTTAATGTAACAACTCTTCTGTCATAGC ATTGTGTGTGGTCCCTATGGACTGTTTACTATCCAA AAGTTCAAGAGAGAAAACAGCACTTGAGGTCTCATC AATTAAAGCACCTTGTGGAATCTGTTTCCTATATTT GAATATTAGATGGGAAAATTAGTGTCTAGAAATACT CTCCCATTAAAGAGGAAGAGAAGATTTTAAAGACTT AATGATGTCTTATTGGGCATAAAACTGAGTGTCCCA AAGGTTTAT TAATAACAGTAGTAGTTA-3', and the sequence of SMAD2-3UTR-mu was: 5'-AGCTTCACCAAT
CAAGTCCCATGAAAAGACTTAATGTAACAACTCTTC TGTCATAGCATTGTGaGaGcTCCCTATGGACTGTTT ACTATCCAAAAGTTCAAGAGAGAAAACAGCACTTGA GGTCTCATCAATTAAAGCACCTTGTGGAATCTGTTT CCTATATTTGAATATTAGATGGGAAAATTAGTGTCT AGAAATACTCTCCCATTAAAGAGGAAGAGAAGAT TTTAAAGACTTAATGATGTCTTATTGGGCATAAAAC TGAGTGTCCCAAAGGTTTATTAATAACAGTAGTAGT TA-3' were also added into the pSI-Check2 vector. SMAD2 plasmid (h-SMAD2-3UTR, Hanbio Biotechnology Co., Ltd.) or its mutated fragments (Fig. S1B) and miR-140-3p were also co-transfected into the $293 \mathrm{~T}$ cell line. Lowercase letters represent the mutation sites. After $48 \mathrm{~h}$ of transfection, a dual-luciferase assay system (Promega Corporation) was used to determine the firefly and Renilla luciferase activities of the transfected cells.

Western blot analysis. After harvesting the chondrocytes from the overcircRNA_0079201 and NC groups, the protein samples were extracted using cell lysis (Tissue cell total protein extraction kit; Applygen Technologies Inc.). The concentration of total protein was evaluated using a BCA assay (Pierce; Thermo Fisher Scientific, Inc.). Subsequently, the protein lysates were separated using SDS-PAGE (Beijing Biosynthesis Biotechnology), transferred onto PVDF membranes and then blocked using skimmed milk (Beijing Solarbio science and Technology, Inc.) for $1 \mathrm{~h}$ at room temperature $\left(22 \pm 3^{\circ} \mathrm{C}\right)$. Following which, the membranes were incubated with anti-Smad2 (1:2,000 dilution; cat. no. ab40855), anti-RUNX2 (1:2,000 dilution; cat. no. ab192256), anti-collagen type $\mathrm{X}$ (COL10A1; 1:2,000 dilution; ab58632), and anti-Indian Hedgehog (1:2,000 dilution; ab39634) (all from Abcam) primary antibodies, overnight at $4^{\circ} \mathrm{C}$. The membrane was then rinsed using 1X TBS-Tween-20 (Beijing Solarbio science and Technology, Inc.) and subsequently incubated for $1 \mathrm{~h}$ at room temperature with a HRP-labeled rabbit anti-mouse $(1: 3,000$ dilution; cat. no. ab6721; Abcam) secondary antibody. Finally, the immunoreactive bands were visualized using an enhanced chemiluminescent kit (Pierce; Thermo Fisher Scientific, Inc.). GAPDH was used as the internal control (1:3,000, cat. no. ab8245; Abcam). If necessary, more than one protein was detected on the same western blot membrane using the WB stripping buffer (cat. no. 21059; Thermo Fisher Scientific, Inc.) and the membrane was probed again with a different primary antibody. ImageJ software (v8.0; National Institutes of Health) was used to measure gray scale values.

In situ hybridization. Human chondrocytes were incubated at $65^{\circ} \mathrm{C}$ for $48 \mathrm{~h}$ with $500 \mathrm{ng} / \mathrm{ml}$ FAM-labeled probe and Cy3-labeled probe (single stranded RNA oligonucleotide probe; hsa-circ-0079201 sequence; 5'-Cy3-GAGCTCTTGGGCCTGG TCTGGGAGGCCTGGCACGCTGGGTGGCTC-3', length 45 bp; hsa-miR-140-3p sequence; 5'-FAM-CCGTGGTTC TACCCTGTGGTA-3', length $21 \mathrm{bp}$ ) according to the manufacturer's instructions (miRCURY LNA miRNA ISH kit; Thermo Fisher Scientific, Inc.), to examine the distribution of hsa_circRNA_0079201 and miR-140-3p expression.

A total of 6,6-week-old C57 mice of either sex (two females mice and four males), with a weight of 17.5-20.5 g were used in the study. The mice was fed using mixed 
Table II. Clinical characteristics of patients with ISS and healthy individuals.

\begin{tabular}{lcc}
\hline Variable & ISS & Healthy controls \\
\hline Sex & & \\
Male & 34 & 39 \\
Female & 42 & 37 \\
Mean age $\pm \mathrm{SD}$, years & $9.03 \pm 2.75$ & $8.38 \pm 2.22$ \\
Age range, years & $4.00-12.3$ & $4.10-12.00$ \\
Mean height $\pm \mathrm{SD}, \mathrm{cm}$ & $120.39 \pm 14.05$ & $132.24 \pm 12.47$ \\
Height range, cm & $87.10-142.2$ & $103.90-151.20$ \\
\hline
\end{tabular}

ISS, idiopathic short stature.

feed (Beijing Keao Xieli Feed Co., Ltd.) at $23 \pm 1^{\circ} \mathrm{C}$, with a light/dark cycle of $12 / 12 \mathrm{~h}$. A total of 12 femur samples from the six mice were extracted once the the six mice had been euthanized with an intraperitoneal injection of $160 \mathrm{mg} / \mathrm{kg}$ sodium pentobarbital and stored in a $4 \%$ formaldehyde solution. Subsequently, the samples were decalcified in 10\% EDTA solution for 4 weeks. Samples were then embedded in paraffin and paraffin-embedded specimens were further cut into 4- $\mu \mathrm{m}$ thick sections for in situ hybridization (miRCURY LNA miRNA ISH kit; Thermo Fisher Scientific, Inc.). The present study was approved by the Animal Ethics Committee of Nanchang University (Nanchang, China).

Von Kossa and alkaline phosphatase (ALP) staining. Formation of mineralized nodules by chondrocytes in vitro was analyzed using Von Kossa staining. The chondrocytes (72 $\mathrm{h}$ post-transfection with the overcircRNA-0079201 vector) were rinsed twice with PBS and fixed in 95\% ethanol solution at room temperature for $10 \mathrm{~min}$. Von Kossa silver solution was used to stain the chondrocytes for $1 \mathrm{~min}$ followed by exposure to ultraviolet light for $10 \mathrm{~min}$ at room temperature $\left(22 \pm 3^{\circ} \mathrm{C}\right)$. The chondrocytes were then mixed with $1 \mathrm{ml}$ Hypo solution for $1 \mathrm{~min}$ at room temperature, after rinsing with distilled water. Subsequently, $1 \mathrm{ml}$ hematoxylin solution was used to stain the chondrocytes for $2 \mathrm{~min}$, followed by $1 \mathrm{ml}$ eosin for $1 \mathrm{~min}$ at room temperature. The calcium nodules exhibited a black/brown or a dark black color and the background presented red.

The cultured chondrocytes cells were washed with PBS three times and fixed with $4 \%$ paraformaldehyde for $15 \mathrm{~min}$ at room temperature. Then, the fixed chondrocytes cells were treated with a 5-bromo-4-chloro-3-indolyl-phosphate/nitro blue tetrazolium AP color development kit (Beijing Solarbio Science and Technology, Co., Ltd.) in the dark for $48 \mathrm{~h}$. The chondrocyte cell lysates were used for detection according to the manufacturer's instructions of the AP assay kit.

Statistical analysis. As aforementioned, quantile normalization and data processing were performed using the Quantile algorithm and 3.11 version of limma packages (https:/www. bioconductor.org/packages/release/bioc/html/limma.html) in R 3.6.0 software (https://www.R-project.org/). Statistical significance was determined using one-way analysis of variance followed by Tukey's post hoc test or with an unpaired t-test between two groups. $\mathrm{P}<0.05$ was considered to indicate a statistically significant difference. All statistical analyses were performed using SPSS (v20.0; IBM Corp.) and GraphPad Prism (v5.0; GraphPad Software, Inc.) software.

\section{Results}

Screening of differentially expressed circRNAs and their miRNA interactions. A total of 152 patients with ISS and healthy individuals were enrolled into the present study, and their demographic and clinical information are summarized in Table II. Among all the individuals, four pairs of patients with ISS and age-/sex-matched controls were screened using circRNA microarray analysis (Fig. 1A). Among the patients with ISS, 145 circRNAs were significantly differentially expressed $(\mathrm{P}<0.05 ; \log \mathrm{FCl}>2.0)$. Compared with that in the normal individuals, the expression levels of 83 and 62 circRNAs were up- and downregulated, respectively, in patients with ISS. Bioinformatics software was used to analyze the enrichment of the 145 differentially expressed circRNAs, as they play an essential role in controlling the transcriptional levels of their parental genes (20-22). The variations in circRNA expression levels between patients with ISS and normal controls were additionally identified using volcano plot filtering (Fig. 1B). The vertical lines correspond to 2-fold up- and -downregulated expression, respectively, and the horizontal line represent $\mathrm{P}=0.05$. The red color represents the upregulated circRNAs and the green color represents the downregulated circRNAs with statistical significance. A scatter plot of the circRNA expression profile was also used to evaluate variations between the two groups (Fig. 1C). The $\mathrm{x}$ - and $\mathrm{y}$-axis in the scatter plot are the mean normalized circRNA signal values ( $\log 2$ scaled). The black FC lines represent 2x FC, therefore the circRNAs lying above and below these black lines displayed $>2.0$-fold up- or downregulation, respectively. The red color represents the upregulated circRNAs and the green color represents the downregulated circRNAs with statistical significance.

circRNA_0079201, but not linear IQCE, is upregulated in ISS and inhibits chondrocyte proliferation and hypertrophy, and reduces mineralization. The top five upregulated circRNAs (hsa_circRNA_0079201, hsa_circRNA_0000423, hsa_circRNA_0051239, hsa_circRNA_0087631 and hsa_ circRNA_0064644), with the highest differential expression values were verified using RT-qPCR, with 76 samples from patients with ISS and normal controls. hsa_circRNA_0079201 expression level was significantly higher in the ISS group compared with that in the control group (Fig. 2A; $\mathrm{P}<0.05$ ), while no significant difference was found in hsa_circRNA_0000423, hsa_circRNA_0051239, hsa_circRNA_0087631 and hsa_circRNA_0064644 between the ISS group and the normal control group ( $\mathrm{P}>0.05)$. IQCE is the host gene of hsa_circRNA_0079201. For verifying the upregulation of hsa_circRNA_0079201, but not linear IQCE, RNase R was used to degrade linear RNA. hsa_circRNA_0079201 expression was not significantly different between the groups with RNase R treatment and the normal control (Fig. 2B). However, linear IQCE was significantly decreased following RNase R treatment (Fig. $2 \mathrm{C} ; \mathrm{P}<0.05$ ). Furthermore, it was observed that 


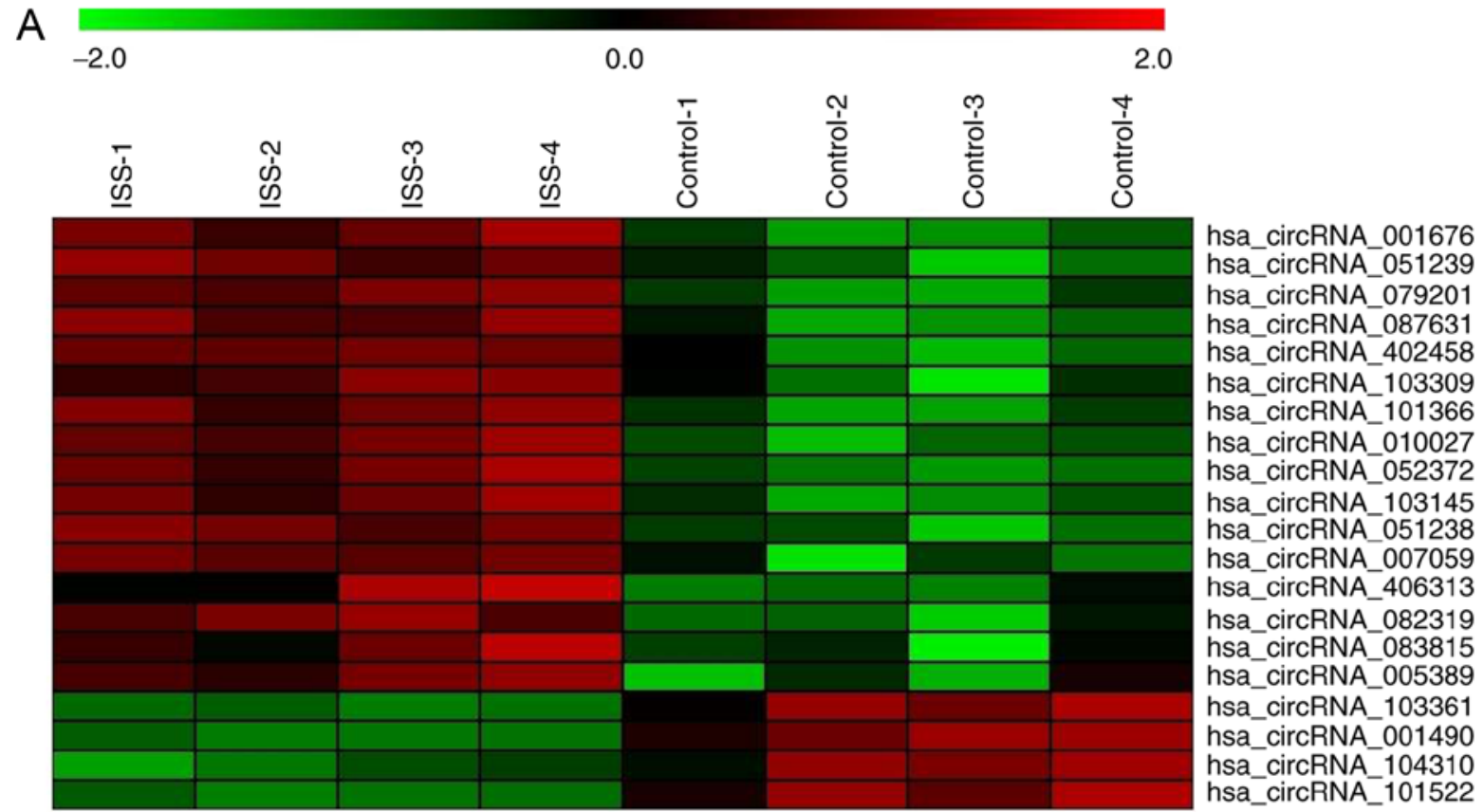

B

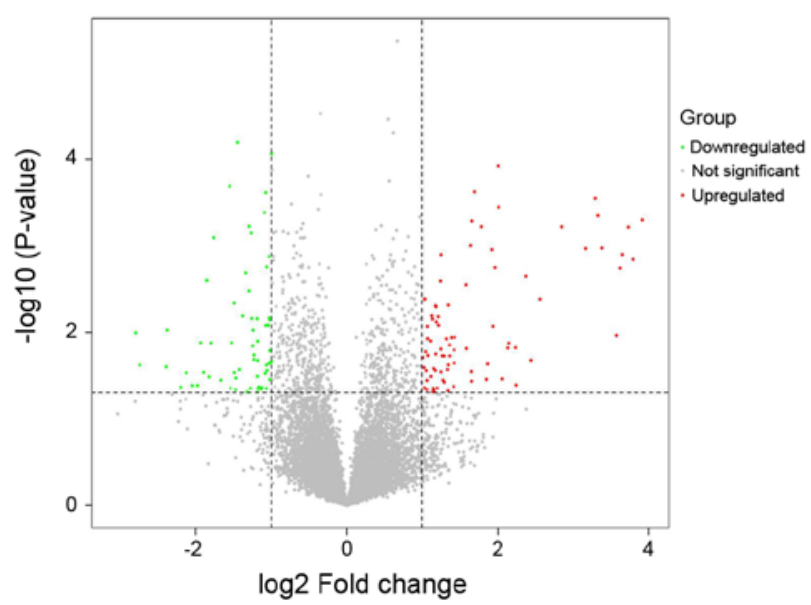

C

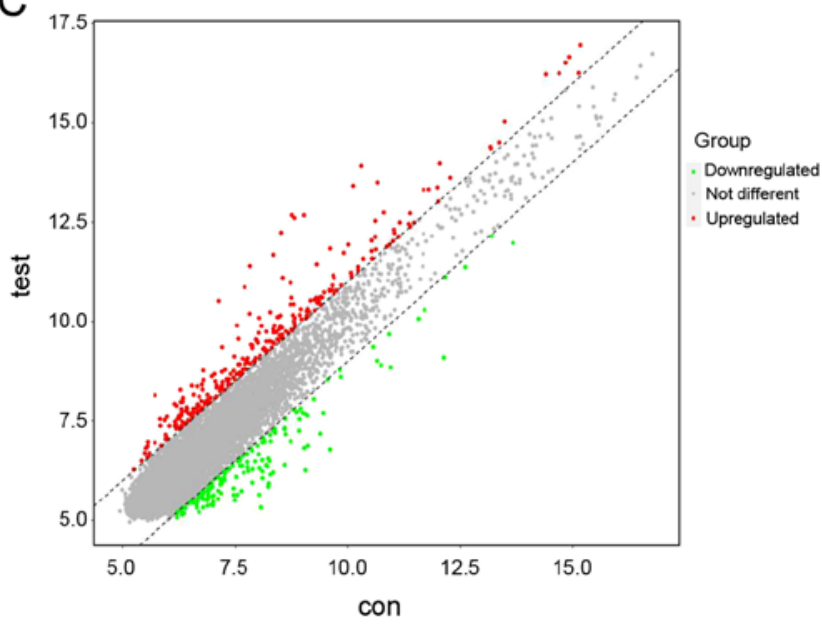

Figure 1. Hierarchical clustering, volcano and scatter plots. (A) Hierarchical clustering of the differentially expressed circRNA in 4 paired patients with ISS and healthy individuals. Red represents the upregulated circRNA and green represents the downregulated circRNA. (B) Volcano plot of the differentially expressed circRNAs. The vertical lines correspond to 2-fold up- and -downregulated, respectively, and the horizontal line represents $\mathrm{P}=0.05$. The red color represents the upregulated circRNAs and the green color represents the downregulated circRNAs with statistical significance. (C) The scatter plot shows the circRNA expression variation between the ISS and normal control. The $\mathrm{x}$ - and y-axis in the scatter plot are the mean normalized circRNA signal values ( $\log 2$ scaled). The black FC lines represent $2 x$ FC, therefore the circRNAs lying above and below these black lines displayed $>2.0$-fold up- or downregulation, respectively. The red color represents the upregulated circRNAs and the green color represents the downregulated circRNAs with statistical significance. circ, circular; ctrl, control.

the expression level of linear IQCE did not exhibit a significant difference between the ISS and the normal control groups (Fig. 2C).

Transfection efficiency was successfully performed, as shown in cells transfected with either overcircRNA_0079201 or the empty plasmid (Fig. S2) and hsa_circRNA_0079201 expression was significantly increased following transfection with overcircRNA_0079201 (Fig. 2D; P<0.05), whilst the CCK-8 and EdU assays revealed that overcircRNA_0079201 significantly inhibited the proliferation of human chondrocytes (Fig. 2E and F; P<0.05). Furthermore, flow cytometry assays revealed that chondrocytes transfected with overcircRNA_0079201 exhibited changes in the cell cycle. As shown in Fig. $2 G$, the number of cells arrested in the $G_{0} / G_{1}$ phase was significantly higher in the pHBLV-hsa_circRNA_0079201 group compared with that in the $\mathrm{NC}$ group $(\mathrm{P}<0.05)$. Furthermore, markedly fewer cells were observed in the $\mathrm{G}_{2} / \mathrm{M}$ and $S$ phases, indicating that $G_{1}$ cell cycle arrest was higher in the overcircRNA_0079201 group compared with that in the negative control group.

To analyze the effect of overcircRNA-0079201 on chondrocyte hypertrophy and mineralization, the mRNA and protein expression level of COL10A1 and RUNX2 was investigated, along with Von Kossa and ALP staining. Significantly lower mRNA and protein expression levels of COL10A1 and RUNX2 were found using RT-qPCR and western blot analysis, respectively, in the overcircRNA-0079201 group (Fig. 3A; $\mathrm{P}<0.05)$. This indicated that overcircRNA-0079201 inhibited 
A

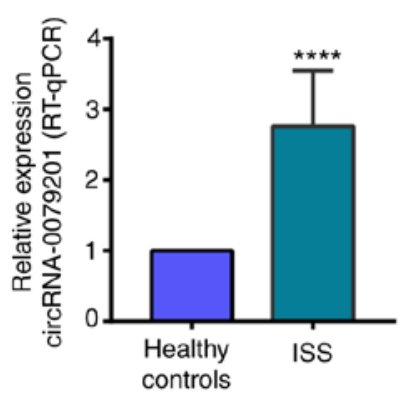

D

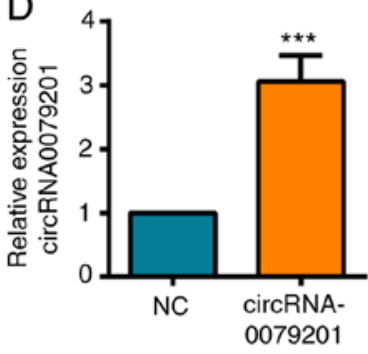

B

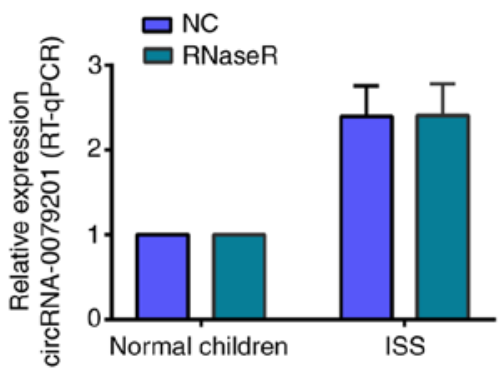

C

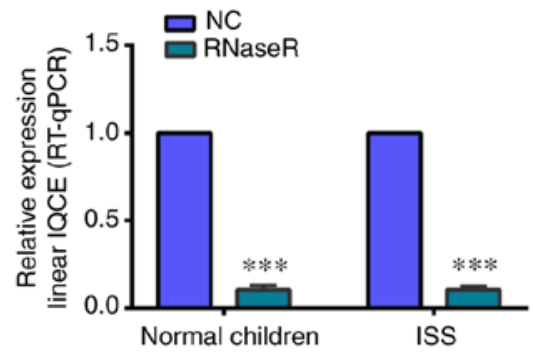

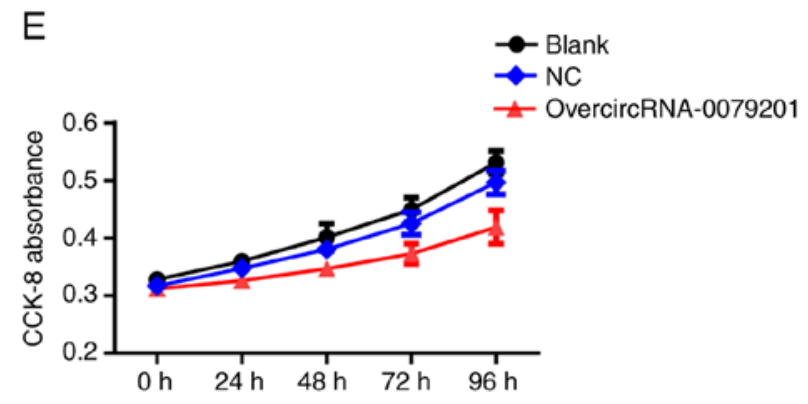
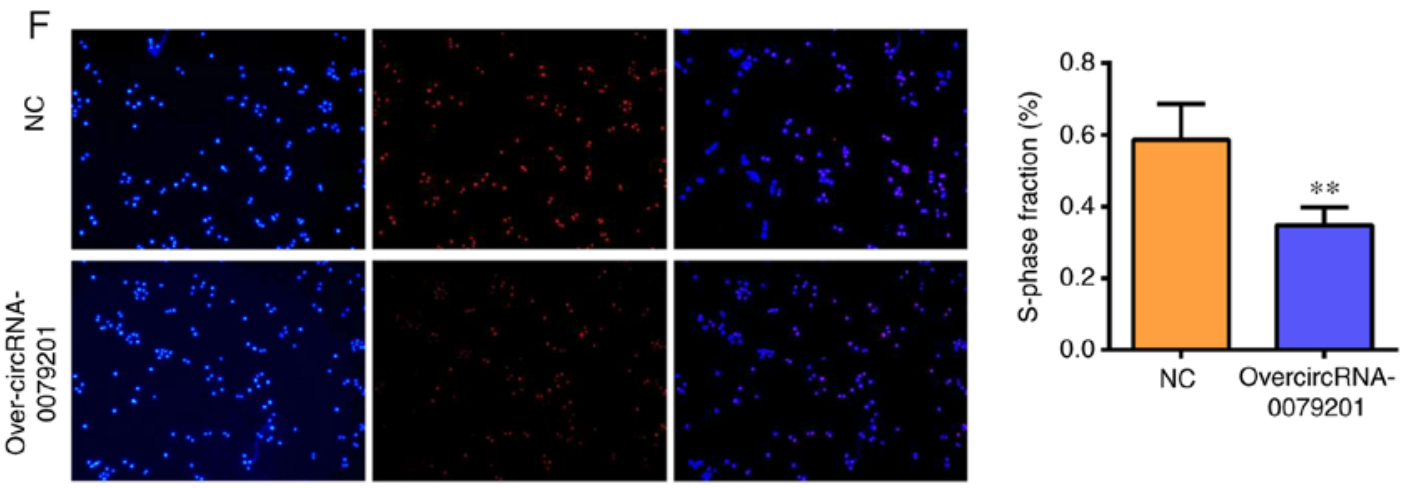

G
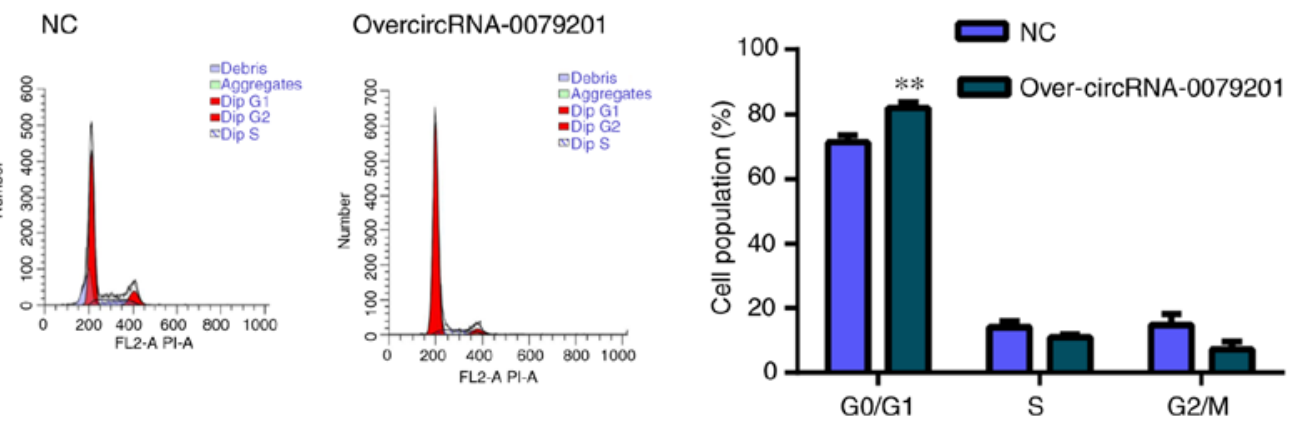

Figure 2.OvercircRNA_0079201 expression significantly decreases human chondrocyte proliferation and causes cell cycle arrest. (A) OvercircRNA_0079201 was confirmed in patients with ISS and normal controls using RT-qPCR. (B) hsa_circRNA_0079201 mRNA expression level was not significantly different between samples treated with RNase R from the patients with ISS and the normal controls. (C) Linear IQCE was significantly decreased following RNase R treatment. (D) hsa_circRNA_0079201 mRNA expression level was significantly increased following transfection with overcircRNA_0079201. (E) The CCK-8 and (F) EdU assays showed that overcircRNA_0079201 significantly suppressed human chondrocyte proliferation. (G) Flow cytometry analysis revealed that the cells transfected with overcircRNA_ 0079201 were arrested in the ${ }_{\mathrm{G} 0 / \mathrm{Gl}}$ phase. The data are presented as the mean $\pm \mathrm{SD}$. $\mathrm{n}=3$. ${ }^{* *} \mathrm{P}<0.01$, ${ }^{* * * *} \mathrm{P}<0.001$ vs. control. ISS, idiopathic short stature; EdU, 5-ethynyl-2'-deoxyuridine; circRNA, circular RNA; IQCE, IQ motif containing E; CCK-8, Cell Counting Kit-8; RT-qPCR, reverse transcription-quantitative PCR.

chondrocyte hypertrophy. Von Kossa staining was an intuitive method to observe the degree of calcification in tissue and cells. As shown in Fig. 3B, Von Kossa staining revealed the reduced mineralization in the overcircRNA-0079201 group. In addition, ALP activity was also reduced in the overcircRNA-0079201 group (Fig. 3C). These results support the hypothesis that over-circRNA_0079201 inhibits chondrocyte proliferation and hypertrophy and reduces mineralization.

miR-140-3p is a hsa_circRNA_0079201 target gene. As some circRNAs can act as a miRNA sponge by binding to target miRNAs and affecting gene expression levels (20-22), 

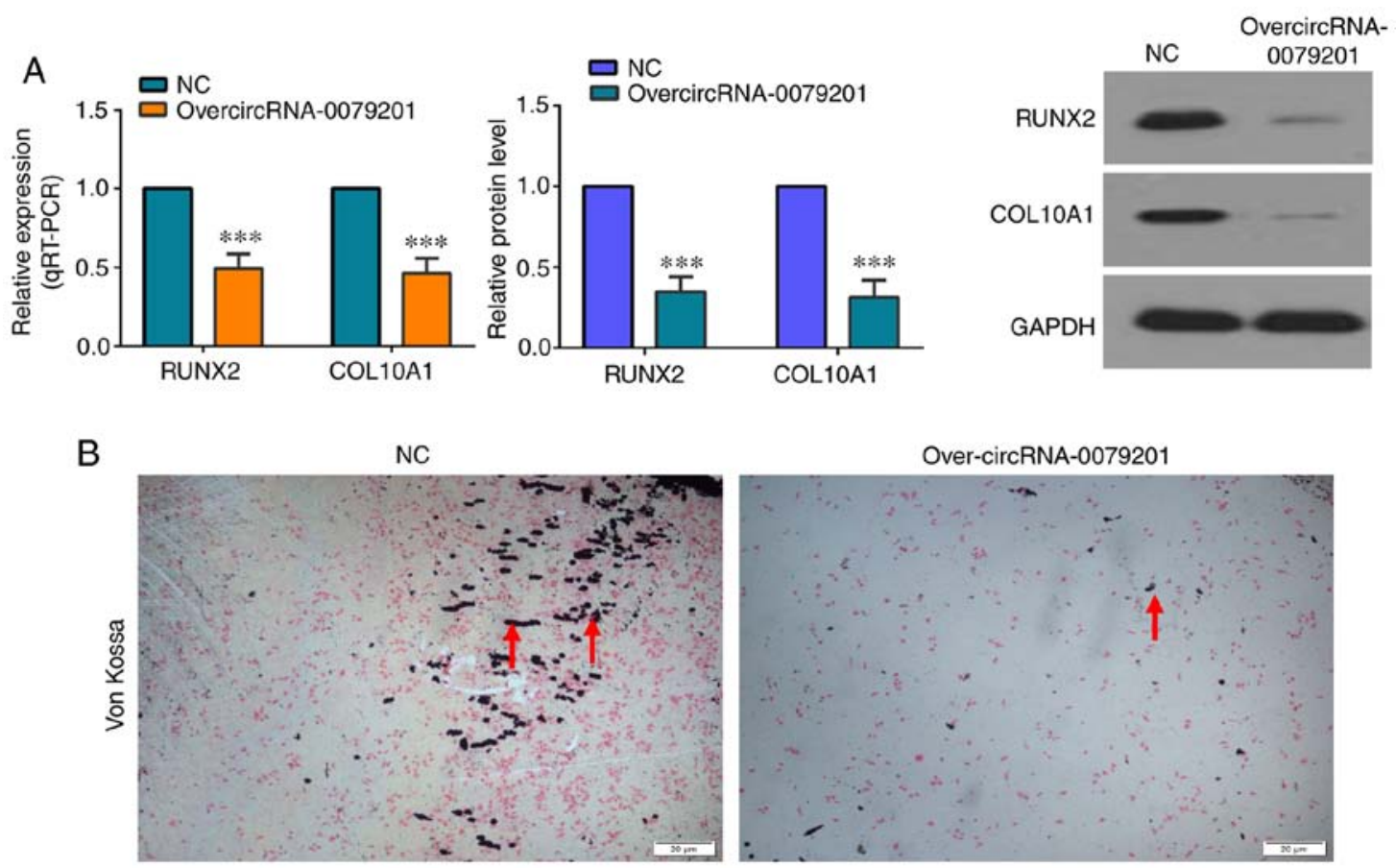

C

NC
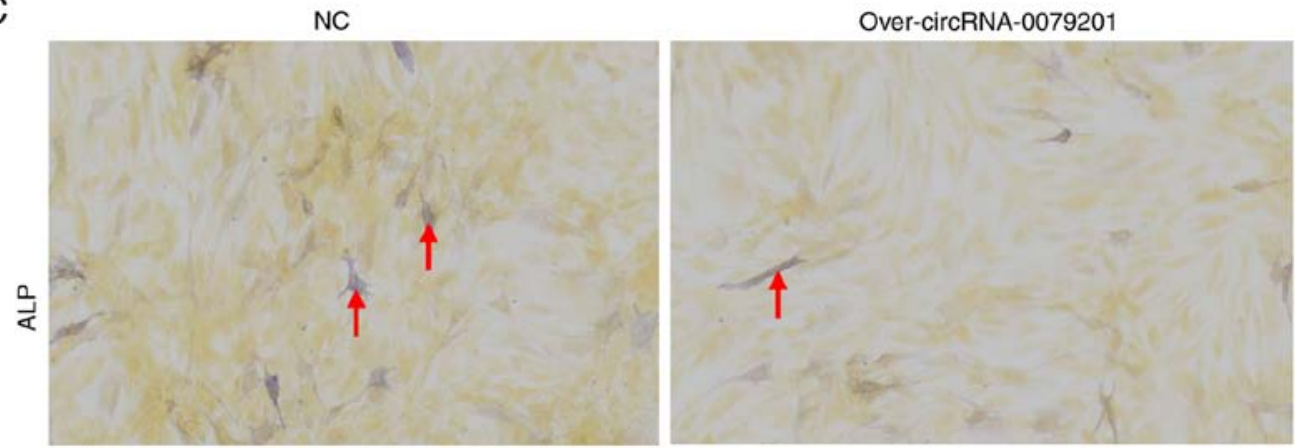

Figure 3. OvercircRNA-0079201 inhibits chondrocyte hypertrophy and endochondral ossification. (A) The protein and mRNA expression level of RUNX2 and COL10A1 was decreased in cells transfected with over-circRNA-0079201, detected using western blot analysis and RT-qPCR, respectively. (B) Von Kossa staining showed the reduced mineralization in cells transfected with overcircRNA-0079201. The positive spots are indictaed using a red arrow. (C) ALP staining revealed that ALP activity was reduced in cells transfected with overcircRNA-0079201. The positive spots are indicated using a red arrow. The data are presented as the mean $\pm \mathrm{SD} . \mathrm{n}=3$. ${ }^{* * *} \mathrm{P}<0.001$ vs. control. RUNX2, runt-related transcription factor 2; ALP, alkaline phosphatase; COL10A1, collagen type X; circRNA, circular RNA; RT-qPCR, reverse transcription-quantitative PCR.

the present study examined the potential interactions between hsa_circRNA_0079201 and miRNAs. The CircInteractome database revealed that hsa_circRNA_0079201 had 13 target genes (hsa-miR-140-3P, hsa-miR-1225-3P, hsa-miR-1236, hsa-miR-1286, hsa-miR-1205, hsa-miR-331-3P, hsa-miR-370, hsa-miR-431, hsa-miR-515-3P, hsa-miR-519e, hsa-miR-532-3p, hsa-miR-564, hsa-miR-578, hsa-miR-581, hsa-miR-635, hsa-miR-636 and hsa-miR-942). The expression level of three of these miRNAs (hsa-miR-140-3P, hsa-miR-635 and hsa-miR-564) was decreased following transfection with overcircRNA_0079201 (Fig. S3). miR-140-3P was selected as a candidate target gene of circRNA_0079201, due to it exhibiting the most significant difference (3.75-fold) (Fig. 4A; $\mathrm{P}<0.05)$. Subsequently, it was found that luciferase activity was reduced in the human chondrocytes co-transfected with wild-type (WT) circRNA_0079201 and miR-140-3p mimic; however, this effect was restored in the cells co-transfected with their mutants (Fig. 4B and C), suggesting that miR-140-3p was a potential target of hsa_circRNA_0079201 in patients with ISS. Notably, in situ hybridization confirmed the co-localization of hsa_circRNA_0079201 and miR-140-3p in human chondrocytes and the neonatal femur growth plate of C57 mice (Fig. 4D).

circRNA_0079201 inhibits chondrocyte proliferation and hypertrophy and reduces mineralization by regulating $m i R-140-3 p$. The transfection efficiency of miR-140-3p mimics is shown in Fig. 5A. The expression of miR-140-3p was upregulated by miRNA mimics 3 days after circRNA_0079201 overexpression via overcircRNA_0079201. Rescue experiments showed that miR-140-3p overexpression reversed the inhibition of human chondrocyte proliferation, hypertrophy and endochondral ossification caused by overcircRNA_0079201 (Fig. 5B-E).

hsa_circRNA_0079201 significantly increases SMAD2 expression by suppressing miR-140-3p. The miR-140-3p target genes were predicted using Starbase database and 

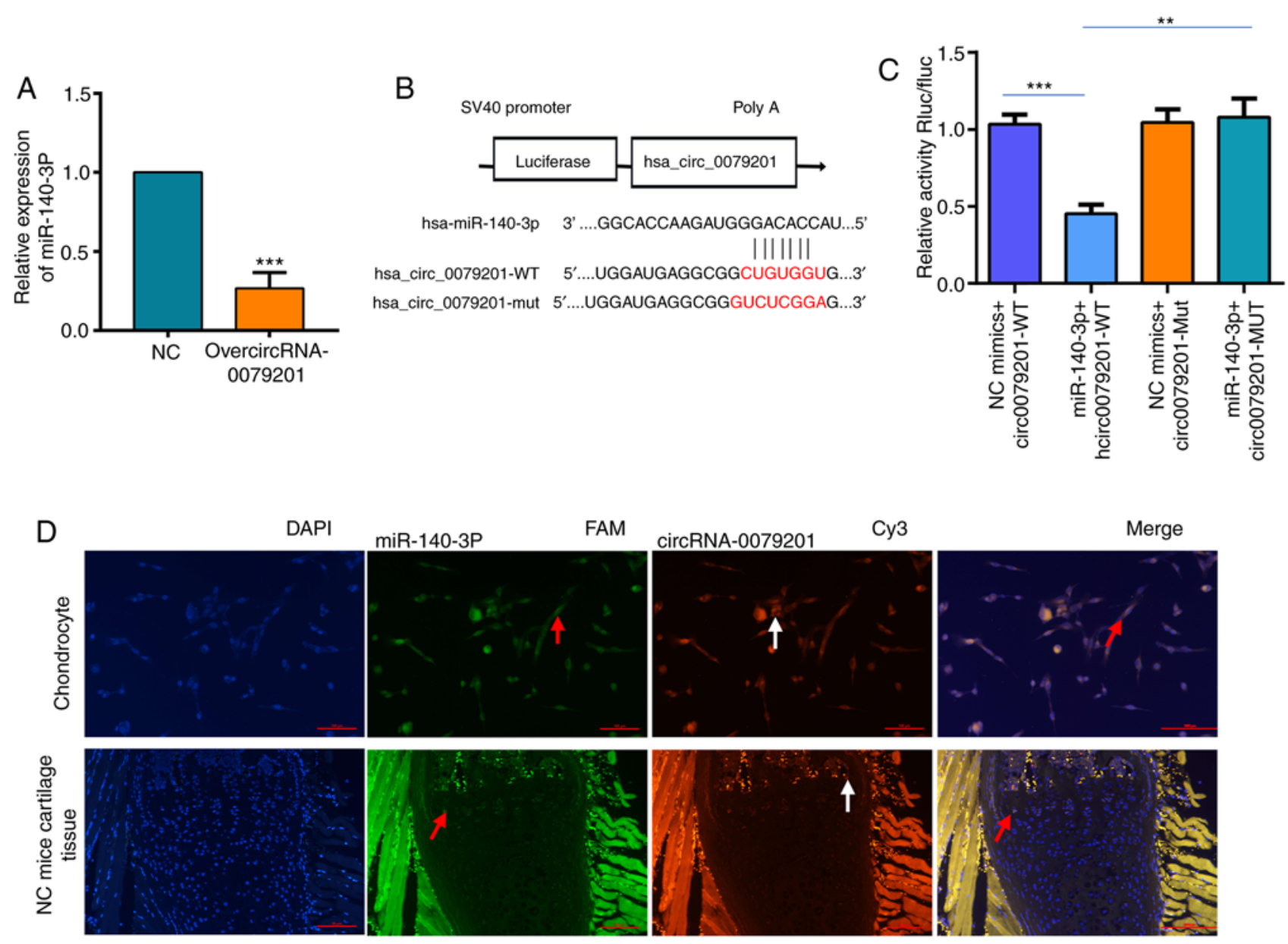

Figure 4. miR-140-3p is a hsa_circRNA_0079201 target gene. (A) miR-140-3p expression was significantly reduced after hsa_circRNA_0079201 was overexpressed in human chondrocytes. (B) The potential target binding sites between miR-140-3p and cirRNA-0079201. (C) Luciferase activity was repressed in human chondrocyte co-transfected with WT hsa_circRNA_0079201 and miR-140-3p mimics, while it was restored in cells co-transfected with Mut hsa_circRNA_0079201 and miR-140-3p mimics. (D) The co-localization of circRNA_0079201 and miR-140-3p in the human chondrocyte and neonatal femur growth plate of $\mathrm{C} 57$ mice was further validated using in situ hybridization. The data are presented as the mean $\pm \mathrm{SD} . \mathrm{n}=3 .{ }^{* * *} \mathrm{P}<0.01,{ }^{* * *} \mathrm{P}<0.001$. WT, wild-type; Mut, mutant; circRNA, circular RNA; miR, microRNA; NC, negative control; Rluc, Renilla luciferase; Fluc, firefly luciferase.

KEGG pathway analyses was performed on these genes, 221 targeted signaling pathways were identified, 110 of which were significantly different $(\mathrm{P}<0.05)$. A total of 4 [TGF- $\beta$, mTOR, Wnt, insulin-like growth factor (IGF) and cell cycle] were associated with chondrocyte development and maturation (Fig. 6A). Notably, SMAD2 participates in three of these pathways (TGF- $\beta$, Wnt and cell cycle) (29-31), and SMAD2 and $\mathrm{miR}-140-3 \mathrm{p}$ were predicted to have three binding sites with high scores by Starbase (Fig. 6B). Therefore, SMAD2 was considered to be a candidate gene in the present study. Luciferase activity was restored in cells co-transfected with SMAD2-Mut and miR-140-3p mimics, and reduced in the 293T cell line co-transfected with SMAD2-WT and miR-140-3p mimics (Fig. 6C and D; $\mathrm{P}<0.05$ ). Meanwhile, SMAD2 was significantly increased compared with that in the control group (Fig. 7A and $\mathrm{B}$; $\mathrm{P}<0.05$ ).

Wang et al (31) confirmed that SMAD2 could inhibit IHH expression and significantly reduced chondrocyte proliferation and hypertrophy in the neonatal growth plate. Therefore, we detected the SMAD2 and IHH expression following transfection with overcircRNA_0079201 vector in the present study. The protein and mRNA expression of SMAD2 was upregulated in the overcircRNA_0079201 group (Fig. 7A and B; P<0.05).
Meanwhile, the expression of IHH, a SMAD2 target gene, presented downregulation compared with that in the control group following transfection with overcircRNA_0079201 vector (Fig. 7A and $\mathrm{B} ; \mathrm{P}<0.05$ ). However, the difference of SMAD2 and IHH expression was not observed between overcircRNA_0079201+ miR-140-3p mimic group and the control group (Fig. 7C and D; $\mathrm{P}>0.05$ ).

\section{Discussion}

ISS is responsible for $50-80 \%$ of children with short stature; however, its pathogenesis remains unclear (32). The GH-IGF-I axis is known to be a vital regulator of skeletal growth during childhood; however, the majority of patients with ISS exhibit no specific abnormalities in this axis (33). Blair and Savage (33) proposed that clinical and molecular scientists should attempt to identify new pathogenetic mechanisms to accurately categorize and treat ISS. Non-coding circRNAs have received increasing attention as novel biomarkers for the diagnosis and treatment of diseases, such as gastric cancer, hepatocellular carcinoma, hypertension, diabetes and rheumatoid arthritis, primarily due to their highly conserved sequences and high stability in mammalian cells compared with that in other non-coding 
A

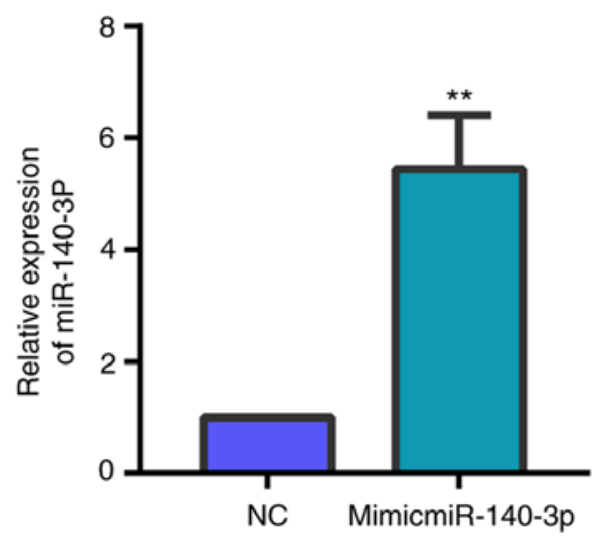

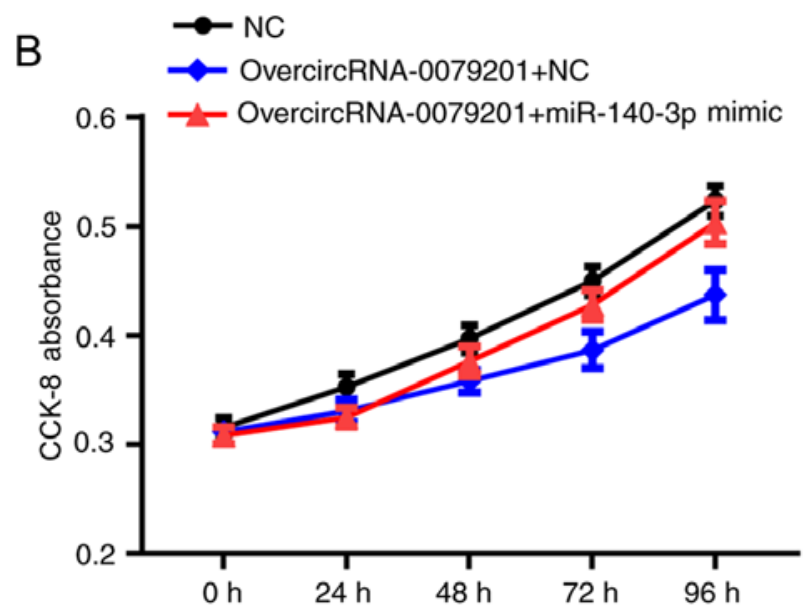

$\square$ NC
OvercircRNA-0079201+NC
$\square$ OvercircRNA-0079201+miR-140-3p mimic
C

NC

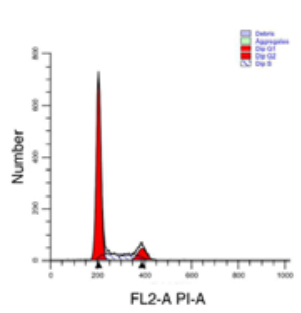

OvercircRNA-0079201+ NC

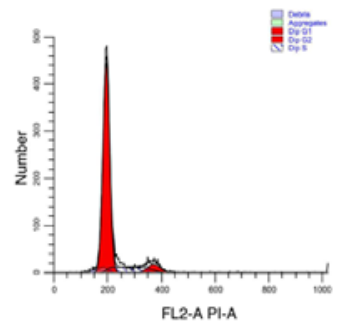

OvercircRNA-0079201+ miR-140-3p mimic

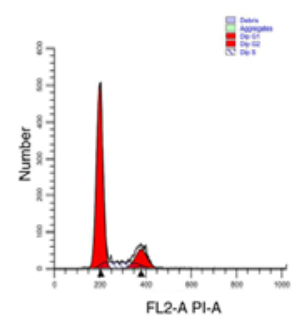

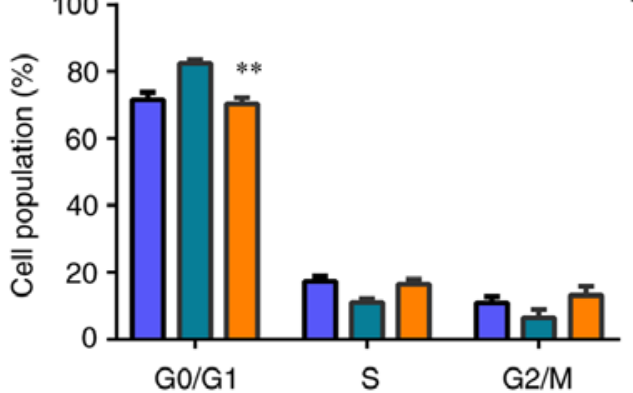

D $\square \mathrm{NC}$

OvercircRNA-0079201+NC

$\square$ OvercircRNA-0079201+miR-140-3p mimic
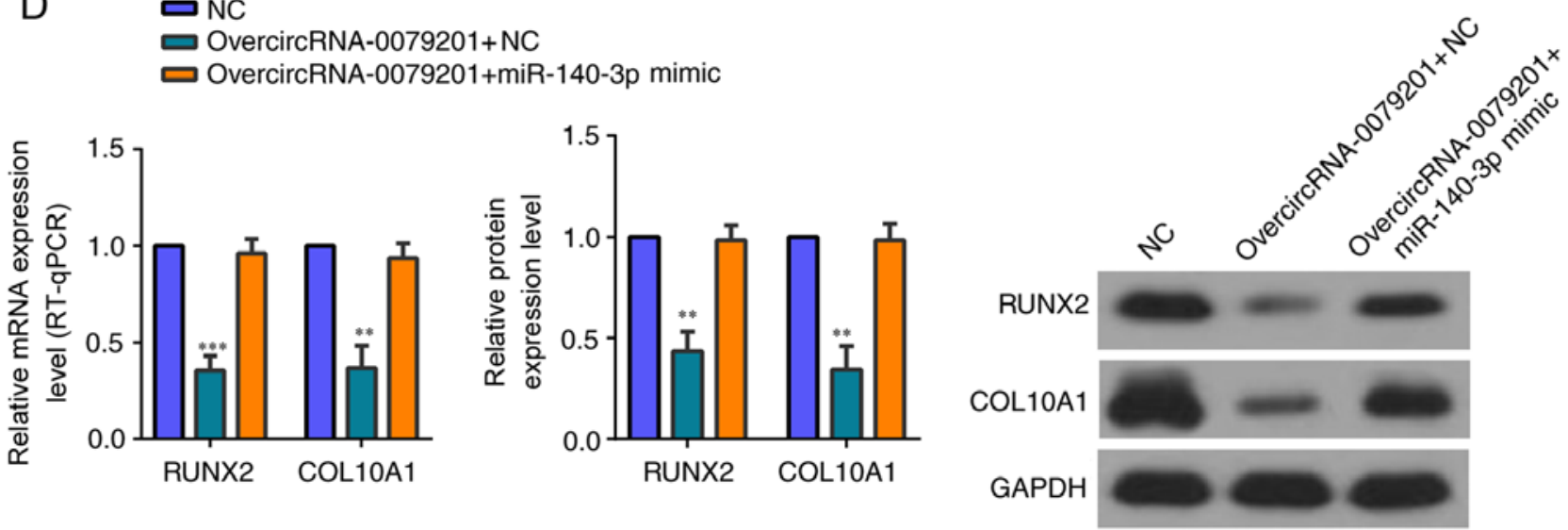

$\mathrm{E}$

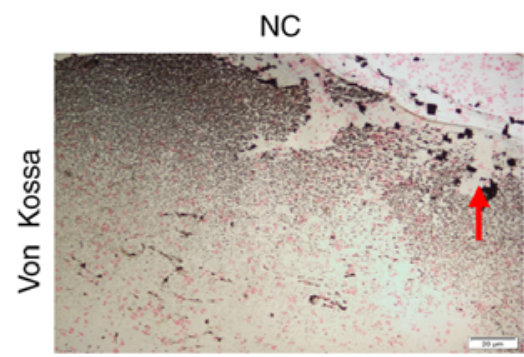

OvercircRNA-0079201+NC

OvercircRNA-0079201+miR-140-3p mimic
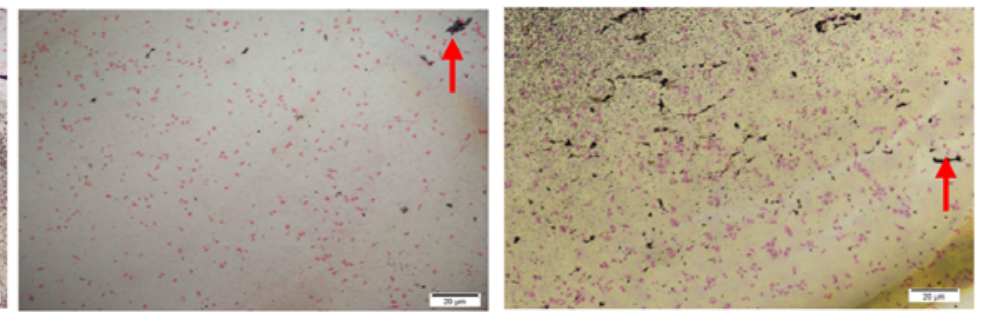

Figure 5. Rescue experiments indicates that miR-140-3p overexpression reversed the inhibition of human chondrocyte proliferation caused by overcircRNA_0079201. (A) The transfection efficiency of miR-140-3p mimics. (B) CCK-8 assay showed that overexpression of miR-140-3p reversed the human chondrocyte decrease in proliferation from the overcircRNA_0079201. (C) There was no significant difference in the number of cells in the different cell cycle phases between cells overcircRNA_0079201 +miR-140-3p and the NC, using flow cytometry. Rescue experiments showed that miR-140-3p overexpression reversed the inhibition of human chondrocyte (D) proliferation and hypertrophy and (E) endochondral ossification caused by overcircRNA_0079201. The data are presented as the mean \pm SD. $n=3 .{ }^{* *} \mathrm{P}<0.01$ vs. control. CCK-8, Cell Counting Kit-8; NC, negative control; circRNA, circular RNA; miR, microRNA; COL10A1, collagen type X; RUNX2, runt-related transcription factor 2. 
A

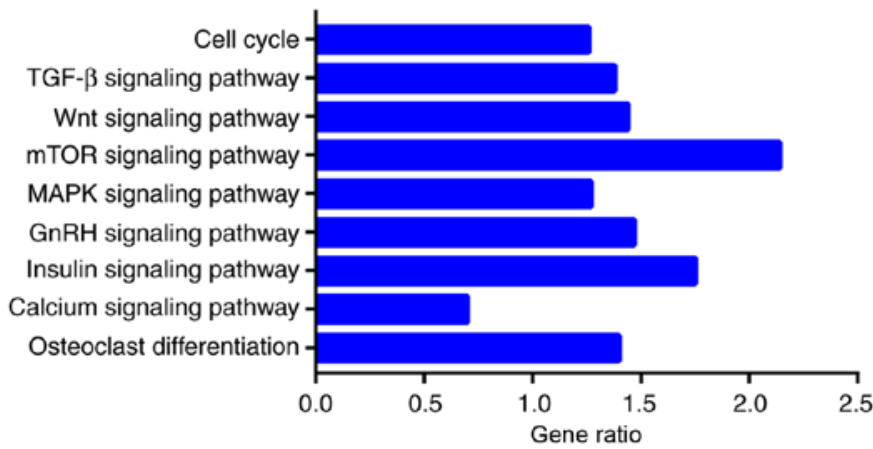

B

Binding site of hsa-miR-140-3p on SMAD2

\begin{tabular}{|c|c|c|}
\hline Binding site & Class & Alignment \\
\hline $\begin{array}{l}\text { chr18:45368129- } \\
\underline{45368134[-]}\end{array}$ & 6 mer & $\begin{array}{l}\text { Target: } 5^{\prime} \text { gucauagcauugugUGUGGUc } 3^{\prime} \\
\qquad|\||\|| \\
\text { miRNA : } 3^{\prime} \text { ggcaccaagaugggACACCAu 5' }\end{array}$ \\
\hline$\frac{\operatorname{chr} 18: 45365363-}{\underline{45365368[-]}}$ & 6 mer & $\begin{array}{l}\text { Target: 5' cccccauuuauaggUGUGGUu } 3^{\prime} \\
\qquad|||| \mid \\
\text { miRNA : 3' ggcaccaagaugggACACCAu 5' }\end{array}$ \\
\hline $\begin{array}{l}\text { chr18:45359792- } \\
\text { 45359797[-] }\end{array}$ & 6 mer & $\begin{array}{l}\text { Target: 5' ugucaaaaguacgaUGUGGUu 3' } \\
\qquad|||| \mid \\
\text { miRNA : } 3^{\prime} \text { ggcaccaagaugggACACCAu 5' }\end{array}$ \\
\hline
\end{tabular}

C

SV40 promoter

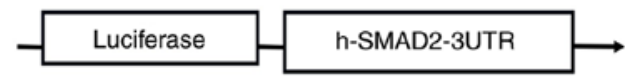

hsa-miR-140-3p $3^{\prime}$ '...GGCACCAAGAUGGGACACCAU...5'

h-SMAD2-3UTR-wt

h-SMAD2-3UTR-mut
| || || |

5' ....UUGUGUGUGGUC... $3^{\prime}$

$5^{\prime}$.... UUGUGAGAGCUC... $3^{\prime}$

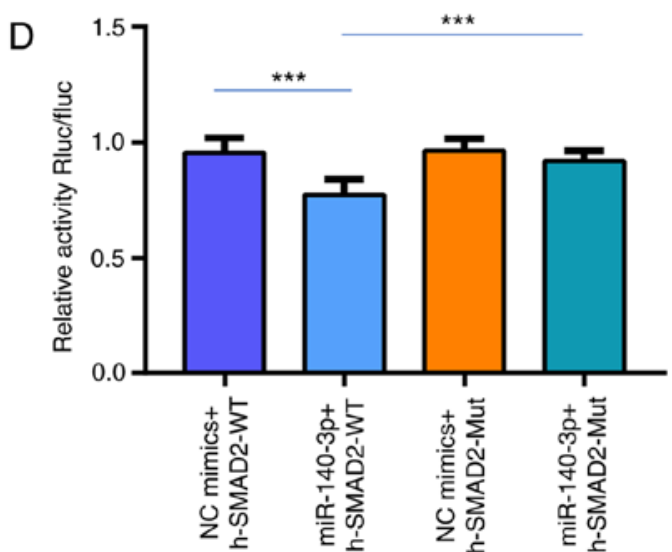

Figure 6. SMAD2 is a target gene of miR-140-3p. (A) miR-140-3p target genes were predicted using Starbase and Kyoto Encyclopedia of Genes and Genomes pathway analyses was performed on these genes, 4 of which (TGF- $\beta$, mTOR, Wnt, and cell cycle) were associated with chondrocyte development and maturation. (B) SMAD2 and miR-140-3p were predicted to have three binding sites with high scores using Starbase. (C) The poteintial target binding site of miR-140-3p and SMAD2. (D) Luciferase reporter assays confirmed that SMAD2 was the target gene of miR-140-3p. The data are presented as the mean \pm SD. $\mathrm{n}=3$. ${ }^{* * *} \mathrm{P}<0.001$ vs. control. SMAD2, SMAD family member 2 ; TGF- $\beta$, transforming growth factor $-\beta$; mTOR, mammalian target of rapamycin; Wnt, wingless/integrated; circRNA, circular RNA; miR, microRNA; WT, wild-type; Mut, mutant; UTR, untranslated region; chr, chromosome; GnRH, gonadotropin releasing hormone.

miRNAs and lncRNAs $(25,34,35)$. Xiong et al (36) analyzed differences in the circRNA expression level in peripheral blood samples from 5 patients with Hashimoto's thyroiditis and 5 healthy volunteers, and identified 627 differentially expressed circRNAs in the patients with Hashimoto's thyroiditis. Upregulated hsa_circ_0089172 expression was confirmed using RT-qPCR, whilst in vitro experiments revealed that hsa_circ_0089172 could be a potential diagnostic biomarker, as it plays a crucial role in the pathogenesis of Hashimoto's thyroiditis by sponging miR-125a-3p. Zhao et al (37), Qian et al (38) and Wang et al (39) assessed differences in the circRNA expression level of peripheral blood samples from patients with community-acquired pneumonia, active pulmonary tuberculosis, and in patients who were small for their gestational age, respectively, with their results suggesting that circRNAs could be reliable biomarkers for diagnosing these conditions. Notably, to the best of our knowledge, the effects of aberrant circRNA expression on ISS has not been investigated, even though an increasing number of studies have investigated the functions of circRNAs in diseases (34-39).

The present study was the first to perform microarray analysis to determine the circRNA expression profiles of patients with ISS and hierarchical clustering to verify the differentially expressed circRNAs. Of the 145 differentially 
A

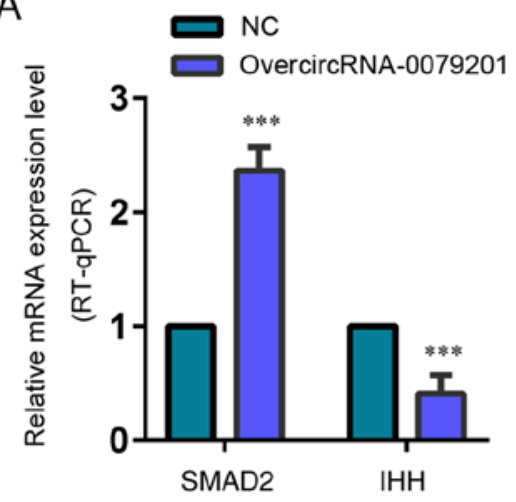

C

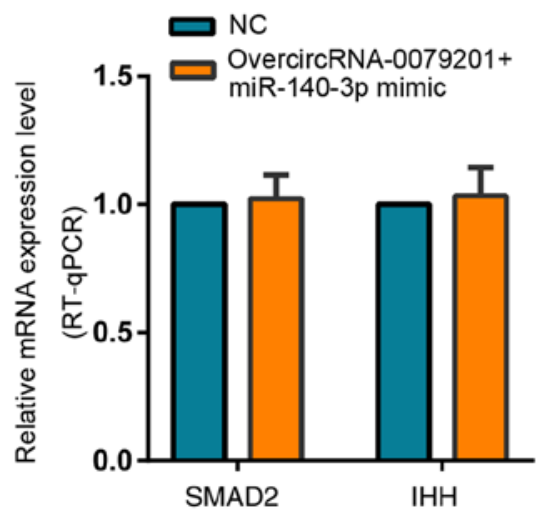

B

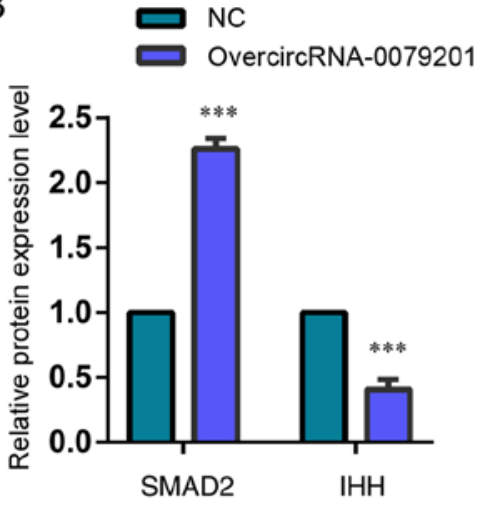

D

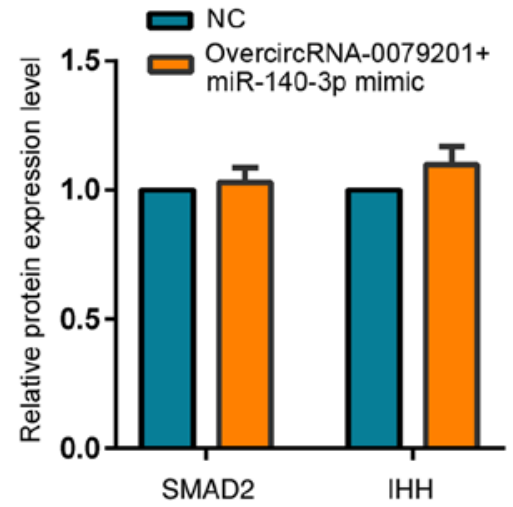

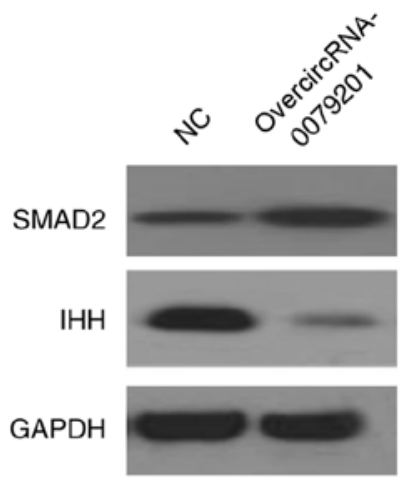

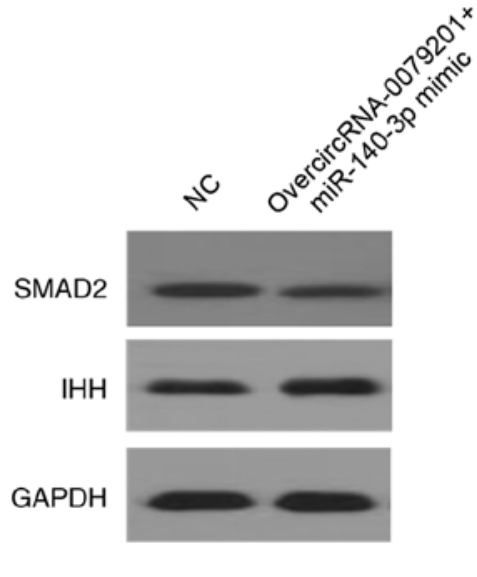

Figure 7. hsa_circRNA_0079201 significantly increases SMAD2 expression by suppressing miR-140-3p. SMAD2 (A) mRNA and (B) protein expression level was increased compared with that in the control group following overcircRNA_0079201. The expression of IHH, a SMAD2 target gene, was decreased compared with that in the control group following overcircRNA_0079201. (C and D) Though SMAD2 showed upregulation in the overcircRNA_0079201 group compared with the normal control group, no significant difference was observed in the SMAD2 mRNA or protein expression levels between the overcircRNA_0079201+ miR-140-3p mimic group and normal control group. Like SMAD2, IHH expression did not also demonstrate significant difference between the overcircRNA_0079201+ miR-140-3p mimic group and normal control group. This indicated hsa_circRNA_0079201 significantly increases SMAD2 expression and decreased IHH expression by suppressing miR-140-3p. The data are presented as the mean \pm SD. $n=3$. ${ }^{* * *} \mathrm{P}<0.001$ vs. control. SMAD2, SMAD family member 2; IHH, Indian hedgehog homolog; NC, negative control; circRNA, circular RNA; miR, microRNA.

expressed circRNAs, 83 and 62 circRNAs were up- and downregulated, respectively in patients with ISS compared with that in the healthy controls. As circRNAs have been found to regulate mRNAs or parental genes (20-22), the biological roles of the ISS-associated circRNAs and their target genes were investigated. hsa_circRNA_0079201 was overexpressed in human chondrocytes to elucidate its role in ISS pathogenesis. As a result, chondrocyte proliferation, hypertrophy and endochondral ossification were significantly suppressed following overcircRNA_0079201. Bioinformatics analysis predicted that miR-140-3p could act as a sponge for hsa_circRNA_0079201, with overcircRNA_0079201 in human chondrocytes inhibiting miR-140-3p expression. Luciferase reporter assays confirmed that miR-140-3p was a target gene of hsa_circRNA_0079201. The co-localization of hsa_circRNA_0079201 and miR-140-3p in the human chondrocyte and neonatal femur growth plate of C57 mice was verified using in situ hybridization. Rescue experiments demonstrated that miR-140-3p overexpression reversed the inhibition of human chondrocyte proliferation, hypertrophy and endochondral ossification caused by overcircRNA_0079201, suggesting that hsa_circRNA_0079201 suppressed chondrocyte, hypertrophy and endochondral ossification via miR-140-3p.
Miyaki et al (17) reported that miR-140-5p null mice, via knockdown experiments, exhibited retarded post-natal growth and short stature phenotypes and that Dnpep was a target gene of miR-140; however, even though miR-140-5p-null mice clearly exhibited a short stature phenotype, Dnpep only had a weak antagonistic effect on bone morphogenetic protein signaling. Therefore, miR-140 may regulate other target genes that inhibit post-natal growth. The present study used Starbase to predict the target genes of miR-140-3p, identifying 221 targeted signaling pathways. A total of 4 pathways (TGF- $\beta$, mTOR, Wnt and cell cycle) were associated with chondrocyte development and maturation according to the literature (29-31). Notably, SMAD2, a member of the SMAD protein family, participates in three of the signaling pathways (TGF- $\beta$, Wnt and cell cycle) (29-31). SMAD proteins are not only signal transducers, but also transcriptional regulators that mediate multiple signaling pathways, such as TGF- $\beta$, BMP, and Wnt signaling pathways (29-31). Wang et al (31) observed that SMAD2 could inhibit IHH mRNA and protein expression levels and significantly reduced chondrocyte proliferation and hypertrophy in the neonatal mouse growth plate. Multiple studies have confirmed that downregulated IHH mRNA and protein expression levels could inhibit chondrocyte 
proliferation and impede longitudinal bone growth $(18,40,41)$; therefore, the present study investigated the role of SMAD2 in ISS. As expected, Starbase identified that SMAD2 and miR-140-3p have three binding sites with a high score, whilst luciferase assays verified that miR-140-3p binds to SMAD2. Following hsa_circRNA_0079201 overexpression in human chondrocytes, miR-140-3p mRNA expression level was found to be decreased, while SMAD2 protein expression level was upregulated. Furthermore, hsa_circRNA_0079201 overexpression decreased the protein expression of IHH, a SMAD2 target gene, in human chondrocytes.

The present study was limited by the fact that it only observed the effects of hsa_circRNA_0079201 on chondrocyte proliferation, hypertrophy and endochondral ossification via the miR-140-3p/SMAD2/IHH pathway in vitro. Therefore, these findings must be confirmed in vivo in WT animals, such as mice or Rhesus monkey's and chondrocyte physiology should be investigated in hsa_circRNA_0079201 overexpression or knockout models. Taken together, these results suggested a novel axis whereby hsa_circRNA_0079201 binds to miR-140-3p to regulate the SMAD2/IHH signaling pathway, thus playing an important role in ISS pathogenesis.

\section{Acknowledgements}

Not applicable.

\section{Funding}

The present study was supported by the National Nature Science Foundation of China (grant no. 81960392) and the project of Science and Technology Department of Jiangxi Province (grant nos. 20161BAB215245 and 20181BAB215019).

\section{Availability of data and materials}

The data used and/or analyzed in this study are available from the corresponding author with reasonable request.

\section{Authors' contributions}

XL conceived and designed the experiments. XL, CY and XD performed the experiments. JJ analyzed the data and wrote the paper. XL, CY and JJ revised the manuscript. All authors approved the final version of the manuscript.

\section{Ethics approval and consent to participate}

Human specimen collection was approved by the Human Research Ethics Committee of the Second Affiliated Hospital of Nanchang University. As all the participants were under the age of 12 years, written informed consent was provided by their parents or legal guardians before their enrolment. In addition, this study was also approved by the Animal Ethics Committee of Nanchang University (Nanchang, China).

\section{Patient consent for publication}

Not applicable.

\section{Competing interests}

The authors declare that they have no competing interests.

\section{References}

1. Cohen LE: Idiopathic short stature: A clinical review. JAMA 311: 1787-1796, 2014.

2. Kang MJ: Novel genetic cause of idiopathic short stature. Ann Pediatr Endocrinol Metab 22: 153-157, 2017.

3. Montalbano A, Juergensen L, Fukami M, Thiel CT, Hauer NH, Roeth R, Weiss B, Naiki Y, Ogata T, Hassel D and Rappold GA: Functional missense and splicing variants in the retinoic acid catabolizing enzyme CYP26C1 in idiopathic short stature. Eur J Hum Genet 26: 1113-1120, 2018.

4. Dias C, Giordano M, Frechette R, Bellone S, Polychronakos C, Legault L, Deal CL and Goodyer CG: Genetic variations at the human growth hormone receptor (GHR) gene locus are associated with idiopathic short stature. J Cell Mol Med 21: 2985-2999, 2017.

5. Hattori A, Katoh-Fukui Y, Nakamura A, Matsubara K, Kamimaki T, Tanaka H, Dateki S, Adachi M, Muroya K, Yoshida S, et al: Next generation sequencing-based mutation screening of 86 patients with idiopathic short stature. Endocr J 64: 947-954, 2017.

6. Kim J, Suh BK, Ko CW, Lee KH, Shin CH, Hwang JS, Kim HS, Chung WY, Kim CJ, Han HS, et al: Recombinant growth hormone therapy for prepubertal children with idiopathic short stature in Korea: A phase III randomized trial. J Endocrinol Invest 41: 475-483, 2018.

7. Heo SH, Choi JH, Kim YM, Jung CW, Lee J, Jin HY, Kim GH, Lee $\mathrm{BH}$, Shin $\mathrm{CH}$, Yoo HW: Comparative proteomic analysis in children with idiopathic short stature (ISS) before and after short-term recombinant human growth hormone (rhGH) therapy. Proteomics 13:1211-1219, 2013.

8. van Gool SA, Kamp GA, Odink RJ, de Muinck Keizer-Schrama SM, Delemarre-van de Waal HA, Oostdijk W and Wit JM: High-dose GH treatment limited to the prepubertal period in young children with idiopathic short stature does not increase adult height. Eur J Endocrinol 162: 653-660, 2010.

9. Cutfield WS and Albert BB: Growth hormone treatment for idiopathic short stature. Pediatr Endocrinol Rev 16 (Suppl 1): S113-S122, 2018.

10. Ying YQ, Hou L, Liang Y, Wu W and Luo XP: Efficacy and safety of recombinant human growth hormone in treating Chinese children with idiopathic short stature. Growth Horm IGF Res 42-43: 80-85, 2018.

11. Michigami T: Regulatory mechanisms for the development of growth plate cartilage. Cell Mol Life Sci 70: 4213-4221, 2013.

12. Lee DS, Roh SY, Choi H and Park JC: NFI-C is required for epiphyseal chondrocyte proliferation during postnatal cartilage development. Mol Cells 43: 739-748, 2020.

13. Chen $\mathbf{J}$ and Long F: mTORC1 signaling controls mammalian skeletal growth through stimulation of protein synthesis. Development 141: 2848-2854, 2014.

14. Beermann J, Piccoli MT, Viereck J and Thum T: Non-coding RNAs in development and disease: Background, mechanisms, and therapeutic approaches. Physiol Rev 96: 1297-1325, 2016.

15. Liu X, She Y, Wu H, Zhong D and Zhang J: Long non-coding RNA Gas 5 regulates proliferation and apoptosis in HCS $-2 / 8$ cells and growth plate chondrocytes by controlling FGF1 expression via miR-21 regulation. J Biomed Sci 25: 18, 2018.

16. Jee YH, Wang J, Yue S, Jennings M, Clokie SJ, Nilsson O, Lui JC and Baron J: mir-374-5p, mir-379-5p, and mir-503-5p regulate proliferation and hypertrophic differentiation of growth plate chondrocytes in male rats. Endocrinology 159: 1469-1478, 2018.

17. Miyaki S, Sato T, Inoue A, Otsuki S, Ito Y, Yokoyama S, Kato Y, Takemoto F, Nakasa T, Yamashita S, et al: MicroRNA-140 plays dual roles in both cartilage development and homeostasis. Genes Dev 24: 1173-1185, 2010.

18. Sun J, Wei X, Li S, Sun C, Wang C, Li P, Wei DL and Wei L: The effects of Indian hedgehog deletion on mesenchyme cells: Inducing intermediate cartilage scaffold ossification to cause growth plate and phalange joint absence, short limb, and dwarfish phenotypes. Stem Cells Dev 27: 1412-1425, 2018.

19. Chen Y, Li C, Tan C and Liu X: Circular RNAs: A new frontier in the study of human diseases. J Med Genet 53: 359-365, 2016. 
20. Greene J, Baird AM, Brady L, Lim M, Gray SG, McDermott R and Finn SP: Circular RNAs: Biogenesis, function and role in human diseases. Front Mol Biosci 4: 38, 2017.

21. Memczak S, Jens M, Elefsinioti A, Torti F, Krueger J, Rybak A, Maier L, Mackowiak SD, Gregersen LH, Munschauer M, et al: Circular RNAs are a large class of animal RNAs with regulatory potency. Nature 495: 333-338, 2013.

22. Ebbesen KK, Kjems J and Hansen TB: Circular RNAs: Identification, biogenesis and function. Biochim Biophys Acta 1859: 163-168, 2016.

23. Haque S and Harries LW: Circular RNAs (circRNAs) in health and disease. Genes (Basel) 8: 353, 2017.

24. Yu CX and Sun S: An emerging role for circular RNAs in osteoarthritis. Yonsei Med J 59: 349-355, 2018.

25. Wang Y, Mo Y, Gong Z, Yang X, Yang M, Zhang S, Xiong F, Xiang B, Zhou M, Liao Q, et al: Circular RNAs in human cancer. Mol Cancer 16: 25, 2017.

26. Aufiero S, Reckman YJ, Pinto YM and Creemers EE: Circular RNAs open a new chapter in cardiovascular biology. Nat Rev Cardiol 16: 503-514, 2019.

27. Lui JC: Regulation of body growth by microRNAs. Mol Cell Endocrinol 456: 2-8, 2017.

28. Livak KJ and Schmittgen TD: Analysis of relative gene expression data using real-time quantitative PCR and the 2(-Delta Delta $\mathrm{C}(\mathrm{T})$ ) method. Methods 25: 402-408, 2001.

29. Kozhemyakina E, Lassar AB and Zelzer E: A pathway to bone: Signaling molecules and transcription factors involved in chondrocyte development and maturation. Development 142: 817-831, 2015.

30. van der Kraan PM, Goumans MJ, Blaney Davidson E and ten Dijke P: Age-dependent alteration of TGF- $\beta$ signalling in osteoarthritis. Cell Tissue Res 347: 257-265, 2012

31. Wang W, Song B, Anbarchian T, Shirazyan A, Sadik JE and Lyons KM: Smad2 and Smad3 regulate chondrocyte proliferation and differentiation in the growth plate. PLoS Genet 12: e1006352, 2016

32. Yang L, Zhang C, Wang W, Wang J, Xiao Y, Lu W, Ma X, Chen L, Ni J, Wang D, et al: Pathogenic gene screening in 91 Chinese patients with short stature of unknown etiology with a targeted next-generation sequencing panel. BMC Med Genet 19: $212,2018$.
33. Blair JC and Savage MO: The GH-IGF-I axis in children with idiopathic short stature. Trends Endocrinol Metab 13: 325-330, 2002.

34. Zhang Z, Yang T and Xiao J: Circular RNAs: Promising biomarkers for human diseases. EBioMedicine 34: 267-274, 2018

35. Arnaiz E, Sole C, Manterola L, Iparraguirre L, Otaegui D and Lawrie CH: CircRNAs and cancer: Biomarkers and master regulators. Semin Cancer Biol 58: 90-99, 2019.

36. Xiong S, Peng H, Ding X, Wang X, Wang L, Wu C, Wang S, $\mathrm{Xu} \mathrm{H}$ and Liu Y: Circular RNA expression profiling and the potential role of hsa circ 0089172 in Hashimoto's thyroiditis via sponging miR125a-3p. Mol Ther Nucleic Acids 17: 38-48, 2019.

37. Zhao T, Zheng Y, Hao D, Jin X, Luo Q, Guo Y, Li D, Xi W, Xu Y, Chen Y, et al: Blood circRNAs as biomarkers for the diagnosis of community-acquired pneumonia. J Cell Biochem 120: 16483-16494, 2019.

38. Qian Z, Liu H, Li M, Shi J, Li N, Zhang Y, Zhang X, Lv J, Xie X, Bai Y, et al: Potential diagnostic power of blood circular RNA expression in active pulmonary tuberculosis. EBioMedicine 27: 18-26, 2018

39. Wang Y, Li SF, Dang YJ, Shi XM, Chen L, Wang N, Cai Y and Zhao YY: Differentially expressed circular RNAs in maternal and neonatal umbilical cord plasma from SGA compared with AGA. J Cell Biochem 121: 713-722, 2020.

40. Deng A, Zhang H, Hu M, Liu S, Wang Y, Gao Q and Guo C: The inhibitory roles of Ihh downregulation on chondrocyte growth and differentiation. Exp Ther Med 15: 789-794, 2018.

41. Karp SJ, Schipani E, St-Jacques B, Hunzelman J, Kronenberg H and McMahon AP: Indian hedgehog coordinates endochondral bone growth and morphogenesis via parathyroid hormone related-protein-dependent and -independent pathways. Development 127: 543-548, 2000.

This work is licensed under a Creative Commons Attribution-NonCommercial-NoDerivatives 4.0 International (CC BY-NC-ND 4.0) License. 